\title{
Análise econômica de dois sistemas de produção de leite no Meio-Oeste catarinense
}

\begin{abstract}
Alisson Perin Zulpo' e Thiago Bernardino de Carvalho²
Resumo - $O$ estudo avaliou a viabilidade econômica de dois sistemas, comparando-os entre si: o sistema compost barn (CB) e o sistema à base de pastos (BP), por meio de simulação, considerando uma superfície agrícola útil idêntica. Os dados foram coletados em Lindóia do Sul, SC, entre setembro de 2018 a maio de 2019, em oito propriedades. Os indicadores elencados na análise foram: valor presente líquido (VPL), taxa interna de retorno (TIR), payback e preço de nivelamento (PN). O sistema CB apresentou VPL de - R\$ 1.391.486,68, e TIR inconclusiva, enquanto o sistema BP gerou um VPL de R\$599.516,04, com TIR de $26,8 \%$. A principal variável para essa discrepância foi o preço do litro do leite, em razão de seu significativo impacto no VPL. Concluiu-se que o sistema CB não apresenta viabilidade econômica para o cenário econômico considerado, ao passo que o sistema BP possui menor risco de investimento e demonstra viabilidade mesmo em cenários econômicos depreciados.
\end{abstract}

Termos de indexação: viabilidade econômica; compost barn; sistema a base de pastos.

\section{Economic analysis of two milk production systems in the Midwest of Santa Catarina}

Abstract - The study evaluated the economic viability of two systems of production: the compost barn (CB) and the system based on grazing (PB), through a simulation, considering a farming surface useful and identical. The data were collected in Lindóia do Sul, between September 2018 to May 2019, in eight farms. The index chosen in the analysis were: net present value (NPV), internal rate of return (IRR), payback and leveling price (PN). The CB system showed NPV of - R\$ 1.391.486,68 and IRR inconclusive, while the BP system has given and NPV of R\$599.516,04, with a IRR of $26,8 \%$. The principal variable for this big difference was the milk liter price, due to its significant impact on NPV. Thus, it is possible to conclude that the CB does not show economic viability to the considerate scenario, on the other hand the PB has lower risks of investments and shows economic viability even in economic depreciated scenarios.

Index terms: economic viability; compost barn; system based on grazing.

\section{Introdução}

O estado de Santa Catarina é atualmente o quinto maior produtor de leite do país, sendo responsável pela produção de 2,97 bilhões de litros em 2018, cujo volume representa $8,78 \%$ da produção nacional (IBGE, 2018), 75\% desta produção origina-se no Oeste Catarinense (EPAGRI/CEPA, 2018).

$O$ modelo de produção de leite catarinense à base de pasto (BP) caracterizase por animais manejados em piquetes, alimentação baseada em pastos e suplementação nutricional, de acordo com o volume de produção leiteira individual das vacas (CÓRDOVA, 2012).

Outro sistema produtivo que vem ganhando notoriedade em Santa Catarina é o sistema Compost Barn, no qual os animais são criados em confinamento, numa instalação com área coletiva para descanso, contendo cama de palha revolvida diariamente e pista de alimentação (RADAVELLI, 2018; COLLINS, 2011).

Pelas características topográficas, climáticas, econômicas e culturais da região, a bovinocultura leiteira passa atualmente por uma fase decisiva, na qual se avalia o sistema produtivo mais adequado a essa região. A decisão do produtor entre um sistema ou outro deve sempre levar em consideração a análise econômica. $\mathrm{O}$ objetivo do estudo foi avaliar a viabilidade de implantação de dois sistemas produtivos de bovinocultura leiteira, na região Meio-Oeste do estado de Santa Catarina.

\section{Material e métodos}

O trabalho foi conduzido no município de Lindóia do Sul, SC, região MeioOeste catarinense. 0 município é o $31^{\circ}$ produtor de leite do Estado, com 24,783 milhões de litros ao ano (IBGE, 2018).

Os dados foram coletados com pesquisa a campo no período de setembro de 2018 a maio de 2019, em oito propriedades, sendo quatro em cada sistema, os quais compreenderam custos fixos e variáveis, plantel leiteiro, produtividade mensal e a estrutura fundiária.

Com os dados médios destas propriedades, simularam-se os dois sistemas produtivos numa estrutura fundiária idêntica representativa da região, iniciando com a implantação até seu retorno econômico projetado no fluxo de caixa para 15 anos.

A propriedade modelo simulada para o estudo possui 25 hectares (ha), sendo 12 ha de superfície agrícola útil. Na simulação a base de pastos, 10ha são destinados à produção de forragem verde e 2 ha à produção de silagem de

Recebido em 3/1/2020. Aceito para publicação em 22/5/2020.

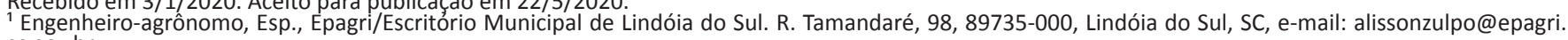

${ }^{2}$ Economista, Dr., CEPEA/ESALQ. Av. Pádua Dias, 11, Pavilhão da Economia, 13418-900, Piracicaba, SP, e-mail: tbcarval@cepea.org.br 
milho. Já no modelo Compost Barn, os 12 ha são destinados à produção de alimento conservado (silagem de milho).

Com cunho de comparação, os sistemas produtivos simulados contemplam apenas a produção de leite, sendo que não foram simulados custos ou receitas com categorias improdutivas. Parte dos índices zootécnicos definidos estão descritos na Tabela 1, e os demais seguem a recomendação de Ferreira \& Miranda (2007).

Nos dois sistemas, a dieta foi balanceada de acordo com o NRC (2001), possibilitando assim, atingir os níveis produtivos almejados.

Na simulação dos investimentos, foram inseridas todas as infraestruturas necessárias à produção leiteira nos dois sistemas, além da aquisição das vacas, conforme Tabela 2. Como receita direta considerou-se a venda do leite e, como indireta, a venda de animais de descarte com base na taxa de reposição adequada.

A análise dos investimentos seguiu critérios de avaliação de rentabilidade na implantação. Para tanto, considerouse os seguintes indicadores: taxa interna de retorno (TIR), valor presente líquido (VPL), payback e preço de nivelamento (PN).

Como se trata de um investimento convencional, o valor presente líquido (VPL) é um fator decisivo para a tomada de decisão sobre qual projeto se torna mais interessante de ser implantado, visto que o mesmo atualiza o retorno econômico esperado pela atividade (PINTO, 2015).

A taxa mínima de atratividade requerida (TMA) definida pelo investidor é de $10 \%$, valor estipulado com base na rentabilidade de títulos de baixo risco (CDBs, títulos públicos), conforme proposto por Souza \& Clemente (2004) e por Casarotto Filho \& Kopittke (2010).

A taxa interna de retorno mostra qual empreendimento é mais adequado, uma vez que reporta o investimento que gera mais patrimônio, acima de uma taxa pré-definida pelo empreendedor. $\mathrm{O}$ período de retorno do capital (payback) demonstra quantos anos seriam necessários para o empreendimento restituir o investimento por intermédio de suas receitas líquidas anuais (KRUGER et al., 2018).

Tabela 1. Dados Zootécnicos admitidos na simulação dos sistemas produtivos Table 1. Zootechnical data admitted in the simulation of productive systems

\begin{tabular}{|lll|}
\hline Índice Zootécnico & À base de pastos & Compost Barn \\
\hline Número de vacas lactantes & 40 & 80 \\
\hline Vacas lactantes (\%) & 83 & 83 \\
\hline Produção individual (I vaca ${ }^{-1}$ dia $^{-1}$ ) & 20 & 35 \\
\hline Raça & Jersey & Holandesa \\
\hline Peso corporal (kg) & 450 & 650 \\
\hline Ingestão de matéria seca (\%) & 4,11 & 4,07 \\
\hline Taxa de reposição & $20 \%$ & $20 \%$ \\
\hline
\end{tabular}

Tabela 2. Capital investido em bens, infraestruturas e semoventes em dois sistemas simulados de produção de leite no Meio-Oeste catarinense, 2019

Table 2. Capital invested in goods, infrastructures and moving in two simulated milk production systems, in the Midwest of Santa Catarina, 2019

\begin{tabular}{lll}
\hline Itens & À base de pastos & Compost Barn \\
\hline Infraestruturas & $\mathrm{R} \$ 94.681,73$ & $\mathrm{R} \$ 389.000,00$ \\
\hline Máquinas e equipamentos & $\mathrm{R} \$ 159.670,19$ & $\mathrm{R} \$ 418.004,00$ \\
\hline Vacas lactantes & $\mathrm{R} \$ 220.000,00$ & $\mathrm{R} \$ 560.000,00$ \\
\hline Vacas secas & $\mathrm{R} \$ 26.860,00$ & $\mathrm{R} \$ 68.000,00$ \\
\hline Total & $\mathrm{R} \$ 501.211,92$ & $\mathrm{R} \$ 1.435 .004,00$ \\
\hline
\end{tabular}

Para demonstrar a volatilidade do mercado do leite, também foram avaliados alguns índices financeiros e sua variabilidade foi simulada com cenários econômicos diversos, principalmente com as flutuações no preço do leite pago ao produtor. Estes índices levantados foram o Custo Operacional Efetivo (COE), Custo Operacional Total (COT), Custo Total (CT) e Lucro Líquido (LL), além da composição dos custos por área de terra explorada e por litro de leite produzido.

O Custo Operacional Efetivo exprime o custo que o produtor tem ao produzir o leite, representando o somatório dos desembolsos com a produção. Já o Custo Operacional Total demonstra a inclusão da mão de obra e depreciação ao COE (MARTIN et al., 1998).

O Custo Total constitui o somatório do COE e do COT, sendo estes configurados pela depreciação de máquinas e equipamentos, custo de oportunidade e outros bens imobilizados (BARROS et al., 2019). No custo de oportunidade da terra, considerou-se o rendimento mé- dio estadual do milho grão por hectare de acordo com a CONAB, (2019a).

Para gerar o fluxo de caixa da atividade, considerou-se a amortização da depreciação na forma linear, prática recomendada em ativos agropecuários, como demonstrado por Barros et al. (2019). No preço do leite, por ser um parâmetro de grande importância, mas oscilante ao longo do tempo (VIANA \& RINALDI, 2010), considerou-se a média do preço do leite recebido pelo produtor (CEPEA, 2019), deflacionada pelo IPCA, numa série histórica de 13 anos, para o estado de Santa Catarina. Como projeção, adotou-se a média de elevação dos preços de acordo com o Relatório Focus (BRASIL, 2019). Nos custos, para esta projeção, adotou-se a média de elevação nos custos de produção de acordo com CONAB (2019b).

\section{Resultados e discussão}

Para o sistema produtivo Compost Barn obteve-se um CT mensal de R\$ 
Tabela 3. Variáveis econômicas mensais resultantes da simulação de dois sistemas produtivos de bovinocultura leiteira em Lindóia do Sul, SC, 2019

Table 3. Monthly economic variables resulting from the simulation of two dairy cattle production systems in Lindóia do Sul, SC, 2019

\begin{tabular}{|c|c|c|}
\hline Indicador & A Base de pastos & Compost Barn \\
\hline $\mathrm{RB}^{(1)}$ & $\mathrm{R} \$ 32.376,00$ & $\mathrm{R} \$ 110.742,00$ \\
\hline $\mathrm{COE}^{(2)}$ & $\mathrm{R} \$ 13.338,41$ & $\mathrm{R} \$ 90.712,82$ \\
\hline $\mathrm{COT}^{(3)}$ & $\mathrm{R} \$ 17.318,35$ & $R \$ 100.235,93$ \\
\hline$C T^{(4)}$ & $\mathrm{R} \$ 17.674,35$ & $\mathrm{R} \$ 100.591,91$ \\
\hline $\operatorname{TIR}(5)$ & $26,81 \%$ & -- \\
\hline VPL $(10 \%)^{(6)}$ & $\mathrm{R} \$ 599.516,04$ & $-R \$ 1.391 .486,68$ \\
\hline Preço de nivelamento $\left(\mathrm{R} \$ \mathrm{I}^{-1}\right)$ & $\mathrm{R} \$ 0,84$ & $\mathrm{R} \$ 1,39$ \\
\hline Preço recebido por litro & $\mathrm{R} \$ 1,26$ & $\mathrm{R} \$ 1,26$ \\
\hline Payback & 3 anos e 9 meses 10 dias & -- \\
\hline Payback descontado & 4 anos 9 meses 23 dias & -- \\
\hline
\end{tabular}

Custo Total (CT); ${ }^{(5)}$ Taxa Interna de Retorno (TIR); ${ }^{(6)}$ Valor Presente Líquido ((VPL)

100.591,91 (Tabela 3) e uma receita bruta total de $\mathrm{R} \$ 110.742,000$. Descontando o CT da RB, somando as receitas não operacionais (descarte de matrizes) e abstraindo os impostos (Imposto de Renda - 27,5\%), obteve-se um lucro

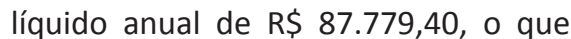
resultou em um VPL negativo de $\mathrm{R} \$$ 1.391.486,68, demonstrando a inviabilidade econômica, fato encontrado também por Barbosa Silveira et al. (2011), trabalhando com animais em free stall.

A alimentação foi o item que mais afetou o COE do sistema $C B$, visto que representou $76,6 \%$, demonstrando alta dependência deste insumo, resultados próximos aos de Lopes \& Magalhães (2005), que demonstraram em seu estudo que a nutrição representou $66,5 \%$ do COE em vacas totalmente confinadas.

Ao avaliar este fator de produção com maior profundidade, os itens mais representativos foram alimento concentrado $(54,9 \%$ do COE) e silagem $(17,6 \%)$.

A nutrição comprometeu $63 \%$ da receita gerada pelo leite, comprovando seu alto impacto financeiro sobre os custos.

O sistema produtivo à base de pastos retornou um VPL positivo de $\mathrm{R} \$$ 599.516,04 (TMA= 10\%), significando que o investimento é mais rentável que uma aplicação financeira com retorno de $10 \%$ ao ano num período de 15 anos.

O sistema à base de pastos gerou $70,69 \%$ menos receita bruta, produziu $71,42 \%$ menos leite, e resultou em $30,6 \%$ mais lucro líquido, quando comparado ao sistema Compost Barn no primeiro ano de comparação, dados estes corroborados por Vilela et al. (1996).

$\mathrm{O}$ sistema à base de pastos mostrouse mais rentável quando comparado ao sistema Compost Barn, por ser um sistema com menor desembolso em alimentação, variável cujo impacto no custo de produção é maior, fato confirmado por
Holmes \& Wilson (1989) e Silva et al. (2008).

O sistema CB não apresentou TIR válida, pois não gerou recursos no Fluxo de Caixa Normal e nem no Fluxo de Caixa Descontado, divergente do sistema $\mathrm{BP}$, que retornou uma TIR de $26,81 \%$ no Fluxo de Caixa Normal e $24 \%$ no Fluxo de Caixa Descontado.

Os custos de produção foram distintos nos dois sistemas produtivos estudados e, em ambos, nota-se o grande impacto do item alimentação. Porém, enquanto no sistema BP o CT de produção por litro foi de $\mathrm{R} \$ \mathbf{0 , 5 8}$, no sistema CB foi de $R \$$ 0,94.

Quando se avaliam os custos de produção por área de terra utilizada observou-se que o sistema CB despendeu $82,42 \%$ mais recursos que o sistema $B P$, e retornou $69,4 \%$ menos lucro líquido, demonstrando que o sistema BP é um mitigante de riscos, visto que emprega menor capital e retorna maior lucro líquido.

Projetando-se cenários econômicos distintos para ambos os sistemas produtivos, nota-se a alta dependência de duas variáveis econômicas: o custo de alimentação diário individual e o preço do leite, sendo esses mais sensíveis que a produtividade diária individual ou mesmo o número de animais ordenhados.

Analisou-se, também, o efeito do preço do leite sobre o VPL (Figura 1) e observou-se que, mesmo em cenários negativos, o sistema BP gera VPL positivo. Já para o sistema CB se tornar viável, é necessária a elevação do preço rece-॰

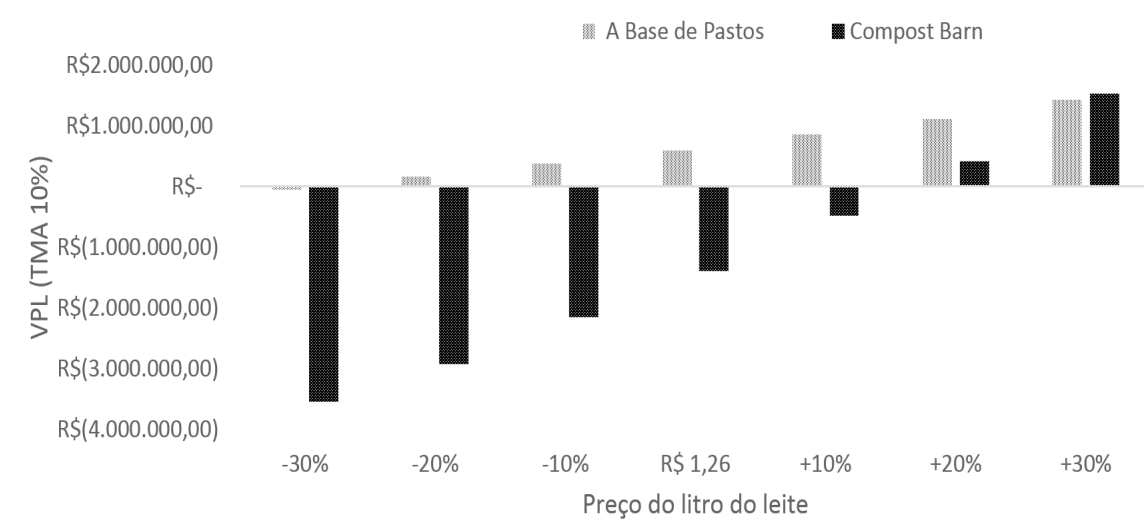

Figura 1. Variação do Valor Presente Líquido (VPL) (TMA10\%) em cenários de elevação e queda do preço do leite pago ao produtor, em dois sistemas produtivos simulados Figure 1. Variation in Net Present Value (NPV) (MRA 10\%) in scenarios of rising and falling milk prices paid to producers in simulated production systems 


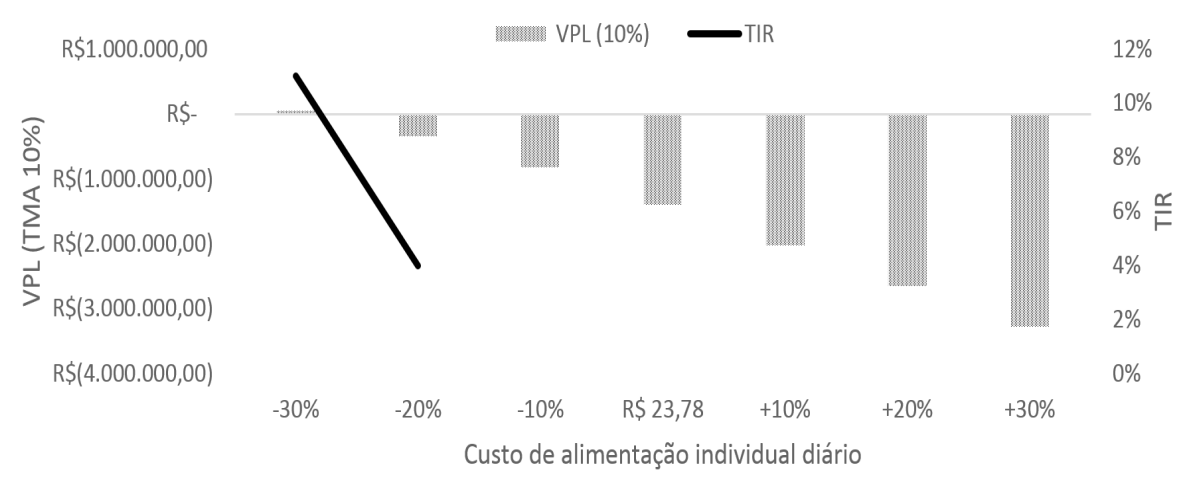

Figura 2. Variação do Valor Presente Líquido (VPL) (TMA10\%) e Taxa Interna de Retorno (TIR) em cenários de elevação e queda do custo de alimentação individual diário, num sistema produtivo de Compost Barn

Figure 2. Variation in Net Present Value (NPV) (MRA 10\%) and Internal Rate of Return (IRR) in scenarios of rising and falling daily individual feeding costs in a Compost Barn production system

bido pelo produtor por litro do leite em, no mínimo, $20 \%$.

Quando se altera o preço do litro do leite em $10 \%$, o produtor do compost barn tem seu VPL alterado em R\$ 905.444,38; já no BP altera-se em R\$ $258.698,40$, mostrando que a sensibilidade ao preço do leite é mais elevada no CB. Conforme destacam os trabaIhos de Galetto (1996) e Marques et al. (2002), em razão de o sistema CB possuir maior produção diária, este poderá obter maior remuneração quando comparado ao sistema BP, porém, só se tornará atrativo economicamente quando o preço superar o valor de $R \$ 1,39$.

Na Figura 2, está demonstrado o efeito do custo de alimentação diário individual no sistema compost barn, com cenários de elevação e redução destes custos. Nota-se que este sistema, nos moldes em que foi simulado, só é viável economicamente com uma redução de $30 \%$ nos custos de alimentação. Há de se considerar que, fazendo esta possível redução, tenderá a não expressar os mesmos níveis produtivos.

Quando se analisa o impacto dos custos diários de alimentação por animal, percebe-se uma discrepância elevada entre $C B(R \$ 23,78)$ e $B P(R \$ 5,18)$, pois no sistema CB grande parte do alimento é importado de fora da propriedade, o que despende mais recursos, principalmente de alimentação e mão de obra, como comprovado por Vilela et al. (1996) e White et al. (2002).

O impacto dos custos de alimentação no sistema BP é pouco representativo. A alteração desse custo em uma

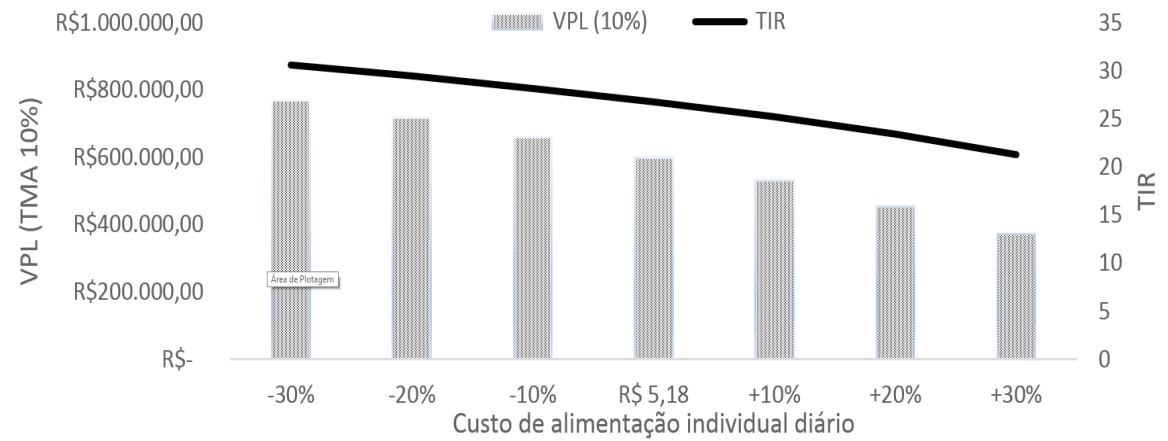

Figura 3. Variação do Valor Presente Líquido (VPL) (TMA10\%) e Taxa Interna de Retorno (TIR) em cenários de elevação e queda do custo de alimentação individual diário, num sistema produtivo à base de pastos, com suplementação

Figure 3. Variation in Net Present Value (NPV) (MRA 10\%) and Internal Rate of Return (IRR) in scenarios of rising and falling daily individual feeding costs in a pasture-based production system, with supplementation dezena percentual altera o VPL (TMA $10 \%$ ) em $\mathrm{R} \$$ 61.419,15 (Figura 3). Sendo assim, somente nos piores cenários econômicos projetados o BP se torna inviável.

\section{Conclusão}

No cenário analisado, o investimento em um sistema Compost Barn é economicamente inviável no Meio-Oeste catarinense, apresentando viabilidade com a elevação do preço do leite em $20 \%$.

$\mathrm{O}$ investimento em um sistema produtivo à base de pastos apresenta viabilidade, retornando mais capital que aplicações financeiras conservadoras.

A variável mais sensível para viabilidade dos projetos é o preço do litro do leite, devido a sua alta influência no VPL ao ser alterado.

\section{Referências}

BARBOSA SILVEIRA, I.D.; PETERS, M.D.P.; STORCH, T.; ZIGUER, E. A.; FISCHER, V. Simulação da rentabilidade e viabilidade econômica de um modelo de produção de leite em free-stall. Arquivo Brasileiro de Medicina Veterinária e Zootecnia, v.63, n.2, p.392398, 2011.

BARROS, G.S. de C.; ALVES, L.R.A.; OSAKI, M.; ADAMI, A.C. de O. Gestão de Negócios Agropecuários com foco no patrimônio. 1. ed. Piracicaba, SP. 120p. 2019.

BRASIL. Banco Central do Brasil. Focus Relatório de Mercado. Disponível em: https://www.bcb.gov.br/publicacoes/focus/26042019. Acesso em mar. 2019.

CASAROTTO FILHO, N.; KOPITTKE, B.H. Análise de Investimentos: matemática financeira, engenharia econômica, tomada de decisão, estratégia empresarial. 11.ed. São Paulo, SP: Atlas, 2010. 432p.

CEPEA. Banco de dados - Preços ao Produtor. Disponível em: https://www. cepea. esalq .usp.br/br/indicador/leite.aspx. Acesso em mar. 2019.

COLLINS, B. L. Viable alternative bedding materials for compost badded pack barns. Kaleidoscope, v.10, p.1-4, 2011.

CONAB. A Cultura do Milho: análise dos custos de produção e da rentabilidade nos anos-safra 2007 a 2017. Disponível em: ht- 
tps://www.conab.gov.br/institucional /publicacoes/compendio-de-studosdaconab/ item/download/15977_22abe6563f37c2 85e7116eb716a $\quad 02804+\& c d=3 \&$ hl=pt $B R \& c t=c|n k \& g|=b r$. Acesso em mar. 2019a.

CONAB. Planilhas de Custos de Produção: Séries Históricas. Disponível em: https:// www.conab.gov.br/info-agro/custos-de-producao/planilhas-de-custo-de-prod ucao/itemlist/category/414-planilhas-de-custos-deproducao-serieshistoricas?start $=20$. Acesso em mar. 2019b.

CÓRDOVA, U. de A. (Org.). Produção de leite à base de pasto em Santa Catarina. Florianópolis, SC, Epagri, 2012, 626p.

EPAGRI/CEPA. Síntese Anual da Agricultura de Santa Catarina 2016-2017. Florianópolis, SC, Epagri. 2018, 200p.

FERREIRA, A.M.; MIRANDA, J.E.C. De. Medidas de Eficiência da atividade leiteira: índices zootécnicos para rebanhos leiteiros, Comunicado Técnico 54. Juiz de Fora, Embrapa. 2007. 8p.

GALETTO, A. Un enfoque preliminar sobre el resultado económico de la intensificación de la produción lechera. Desarollo Rural, Información Técnica n.58 - EEA Rafaela-INTA, 1996.

HOLMES, C.W.; WILSON, G.F. Produção de leite a pasto. Instituto Campineiro de Ensino Agrícola. Campinas, SP. 1989. 708p.

IBGE. Pesquisa Pecuária Municipal - PPM 2018. Disponível em: https://ibge.gov. br/ estatisticas/economicas/agricultura-epecuaria/9107-producao-da-pecuaria-munici pal.html?=\&t=resultados. Acesso em: dez. 2019.

KRUGER, S.D.; BOTTINI, M.A.; DIEL, F.J.; GOLLO, V. Análise de viabilidade do retorno econômico-financeiro das atividades leiteira e avícola. In: CONGRESSO BRASILEIRO DE CUSTOS, 25, 2018. Anais[...], Vitória, ES, 2018.

LOPES, M. A.; MAGALHÃES, G. P. Análise da rentabilidade da terminação de bovinos de corte em confinamento: um estudo de caso. Arquivo Brasileiro de Medicina Veterinária e Zootecnia, v.57, p.374-379, 2005.

MARQUES, V.M.; REIS, R.P.; SÁFADI, T.; REIS, A.J. Custo e escala na pecuária leiteira: estudo de casos em Minas Gerais. Ciência e Agrotecnologia, v. 26, p. 1027-1034, 2002

MARTIN, N.B.; SERRA, R.; OLIVEIRA, M.D.M.; ÂNGELO, J.A.; OKAWA, H. Sistema Integrado de Custos Agropecuários: Custagri. Informações Econômicas. v. 28. n.1. São Paulo, SP, 1998.

NATIONAL RESEARCH COUNCIL - NRC. Nutrient requirements of dairy cattle. 7.rev.ed. Washington, D.C.: 2001. 381p.

PINTO, A.L.M. Ferramenta de gestão na pecuária leiteira: análise do investimento em melhorias para o bem-estar de vacas. $150 f$. Dissertação (Mestrado em Ciências) - Escola Superior de Agricultura "Luiz de Queiroz", Universidade de São Paulo, Piracicaba, SP, 2015.
RADAVELLI, W.M. Caracterização Do Sistema Compost Barn Em Regiões Subtropicais Brasileiras. 90f. Dissertação (Mestrado em Zootecnia). Universidade do Estado de Santa Catarina, Chapecó, SC, 2018

SILVA, H.A. DA; KOEHLER, H.S.; MORAES, A. DE; GUIMARÃES, V.D.; HACK, E.; CARVALHO, P.C. de F.; Análise de Viabilidade Econômica da produção de leite a pasto e com suplementos nas regiões dos Campos Gerais Paraná. Ciência Rural, v.38 n.2, p. 445-450. 2008.

SOUZA, A.; CLEMENTE, A. Decisões financeiras e análise de investimentos. São Paulo, SP, Atlas, 2004. 200p.

VIANA, G.; RINALDI, R.N.; Principais fatores que influenciam o desempenho da cadeia produtiva do leite - um estudo com os produtores de leite do município de Laranjeiras do Sul/PR. Organizações Rurais \& Agroindustriais, v.12, n.2, p. 263-274, 2010.

VILELA, D.; ALVIM, M.J.; CAMPOS, O.F.; RESENDE, J.C. Produção de leite de vacas Holandesas em confinamento ou em pastagem de coast-cross. Revista da Sociedade Brasileira de Zootecnia, v.25, n.6, p.1228-1244, 1996.

WHITE, S.L.; BENSON, G.A.; WASHBURN, S.P.; GREEN, J.T. JR. Milk Production and Economic Measures in Confinement or Pasture Systems Using Seasonally Calved Holstein and Jersey Cows. Journal Dairy Science, v.85, p.95-104. 2002 .

\section{Não deixe sua consciência escorrer pelo ralo: preserve a água e evite o desperdício.}

